\title{
BMJ Open Dialogues between nurses, patients with heart failure and their partners during a dyadic psychoeducational intervention: a qualitative study
}

\author{
Maria Liljeroos, ${ }^{1,2}$ Susanna Ågren, ${ }^{1,3}$ Tiny Jaarsma, ${ }^{4}$ Anna Stromberg ${ }^{1,5,6}$
}

To cite: Liljeroos M, Ågren S, Jaarsma T, et al. Dialogues between nurses, patients with heart failure and their partners during a dyadic psychoeducational intervention: a qualitative study. BMJ Open 2017;7:e018236. doi:10.1136/ bmjopen-2017-018236

- Prepublication history for this paper is available online. To view these files, please visit the journal online (http://dx.doi. org/10.1136/bmjopen-2017018236).

Received 16 June 2017 Revised 8 August 2017 Accepted 9 August 2017

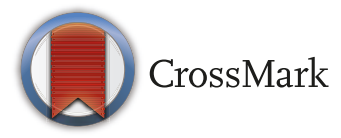

${ }^{1}$ Department of Medicine and Health Sciences, Linköping University, Linköping, Sweden ${ }^{2}$ Centre for Clinical Research Sörmland, Uppsala University, Eskilstuna, Sweden

${ }^{3}$ Department of Cardiothoracic Surgery, Linköping University, Linköping, Sweden

${ }^{4}$ Department of Social and Welfare Studies, Linköping University, Linköping, Sweden ${ }^{5}$ Department of Cardiology, Linköping University, Linköping, Sweden

${ }^{6}$ Sue and Bill Gross School of Nursing, University of California Irvine, Irvine, USA

Correspondence to

Dr Maria Liljeroos;

maria.liljeroos@hotmail.com

\section{ABSTRACT}

Objectives To describe nurses' documentation of the content in a psychoeducational intervention inspired by Stuifbergen's model addressing cognitive, supportive and behavioural needs of patient-partner dyads affected by heart failure.

Design A descriptive qualitative design was used analysing nurses' documentation in a dialogue guide based on a health promotion model.

Settings The dialogue guide was used during three nurse-led sessions at two heart failure clinics in Sweden with patients affected with heart failure and their partners during the years 2005-2008.

Participants The dialogue guides from 71 patient-partner dyads were analysed using direct deductive content analyses. Patients' mean age was 69 years and $31 \%$ were female, partners' mean age was 67 years and $69 \%$ were female.

Results The findings supported the conceptual health promotion model and identified barriers, recourses and self-efficacy described by the dyads within each category. Conclusion The dyads described that during the sessions, they had gained enhanced knowledge and greater confidence to handle their life situation and expressed that they needed psychoeducational support during the whole illness trajectory. The results may guide and help to improve content and quality when caring for patients affected with heart failure and their partners and also when designing new interventions.

Trial registration number NCT02398799; Post-results.

\section{INTRODUCTION}

Heart failure (HF) is a serious condition and the leading cause for hospitalisation, and readmissions for deterioration remain high. Despite most patients receiving education on how to manage the disease, many are not able to adequately engage in self-care activities. ${ }^{2}$

$\mathrm{HF}$ is characterised by a number of signs and symptoms that influence both the patients' and their partners' life situation. Thus, being supported by a partner is important and could improve patients' ability to perform self-care. ${ }^{34}$ Partners might need support from healthcare to be able to help the patient handle self-care in

\section{Strengths and limitations of this study}

- A strength of this study is that the patient-nurse discussions were structured on an existing health promotion model.

- This study adds knowledge on how a health promotion model could be used when delivering a psychoeducational intervention to patients with heart failure and their partners.

- Analysis of nurses' documentation did not allow for consideration of broader contextual factors related to patient-partner cognitive, supportive and behavioural needs and therefore future in-depth qualitative research could give further insights.

everyday life..$^{5-8}$ If the partner lacks knowledge of HF or does not understand how to support the patient, then giving support to the patient may be difficult. Interventions to improve HF self-care should therefore also include involvement of the patient's partner and family.

However, more responsibilities laid on partners can lead to increased physical and mental distress, which in turn can cause caregiver burden. ${ }^{9} 10$ Previous studies have found that as much as $30 \%$ of the partners perceive a moderate caregiver burden. ${ }^{11} 12$

Guidelines state that education is crucial for patients with HF and it is also advised to include partners in the educational programme. ${ }^{1}$ The topics included in the nurse's educational sessions are recommended to cover various aspects of self-care and actions to take should symptoms worsen. Since patient health outcomes are related to the quality of communication with health professionals, ${ }^{13}$ teaching strategies should be tailored to suit each patient and reflect the patient's knowledge, motivation and cognitive function. ${ }^{14}$

Patients and partners wish for more support from healthcare professionals to be able to handle their situation. To 
date, contemporary HF educational programmes are mainly patient focused, but recently awareness of partners' psycho-educational needs has been increasing. ${ }^{5}$ However, there are still few HF interventions addressing both patients' and partners' educational needs. A recent review searched for effective education for HF patients and informal caregivers and found six studies with wide variation in quality. ${ }^{15}$ The authors requested further theory-driven interventions to demonstrate which family-based HF interventions are effective for HF patients and informal caregivers.

\section{BACKGROUND}

A few studies have evaluated the effects of family focused, educational interventions and the results give no clear guidance on how programmes should be designed to give the best effect on patient and partner outcomes. ${ }^{1516}$ One intervention improved both patients' and partners' knowledge but showed modest improvements in other outcomes. ${ }^{17}$ Two studies found that caregiver depression or anxiety did not differ between the educational and control groups over time. ${ }^{18}$ Caregiver burden has shown dissimilar results, one supportive intervention study found burden reduced after 3 months for partners in the intervention group, ${ }^{19}$ whereas another study found no group difference after 24 months. ${ }^{20}$ Only one previous study was guided by an educational theory, ${ }^{17}$ using self-determination theory ${ }^{21}$ to guide HF patients and informal caregivers on decreasing sodium intake.

The lack of conclusive research inspired us to develop a structured dialogue guide based on a conceptual health promotion model constructed by Stuifbergen et $a l^{22}$ delivered during three nurse-led psychoeducational sessions at the HF outpatient clinic. When the study was designed, there were no previous theory-based studies in the HF population; the model used was originally developed and tested for patients with multiple sclerosis (MS). MS is a disease with a path similar to HF, with the burden of fatigue and decreased physical ability. The model focuses on promoting health and not solely helping individuals to manage a disease and propose that health promoting behaviours are influenced by a person's perceived barriers, resources and self-efficacy for health behaviours. The concepts in the model are developed from Pender's model of health promotion ${ }^{23}$ and Bandura's self-efficacy theory. ${ }^{24}$ The assumption is that development of knowledge and skills reduces barriers and enhances resources and self-efficacy. This will result in better health promoting behaviours, such as self-care. Self-efficacy can be defined as an individual's belief in his or her ability to succeed in a particular situation. ${ }^{24}$ Individuals with high self-efficacy have been found to be more likely to view perceived difficulties, such as self-care activities, as something to be mastered rather than something to avoid. ${ }^{25}$ Gaining knowledge about disease management is viewed as the basis for the choice of behaviours to sustain and enhance quality of life. ${ }^{24}$
A previous intervention programme designed within this model had a cognitive component delivering information, but the major component focused on improving patient's self-efficacy by identifying barriers, enhancing resources, increasing support and helping to develop skills and beliefs in one's own capacity to achieve desired behaviour changes. The programme included lifestyle change classes for 8 weeks and telephone follow-up for 3 months. The results showed positive effects regarding health behaviours, health-promoting behaviours, mental health and pain. ${ }^{22} 26$

The model had not previously included partners or families, but as partners provide a lot of support to patients' self-care, here the model was used for the patient-partner dyad. The caregiving situation may be considered as a stressor that poses demands and is often perceived as a stressful or challenging situation for both the partner providing care and the patient receiving care. ${ }^{27}$ Caregiving can be conceptualised as a dyadic interpersonal interaction that involves both the caregiver's and the patient's perspectives. ${ }^{27}$

The current intervention focused on problem solving, information acquisition, self-care management, and emotional and social support to dyads. The nurses delivering the intervention assisted the dyads in recognising and modifying factors that contributed to physical and emotional distress, by supporting them in changing their thoughts and rooted behaviours and implementing strategies for HF self-care management and maintenance.

The dialogue guide was used as a base for the conversations during three sessions and included practical, educational and psychosocial topics that were addressed during the nursing sessions. The result of the interventional study is reported elsewhere. ${ }^{20}$ In this study, we set out to analyse the nurses' documentation from the psychoeducational sessions. This was done to see how the Stuifbergen, Becker $^{22}$ model was used during the psychoeducational intervention provided by nurses to the patient-partner dyads. We also wanted to obtain a deeper understanding of what topics the dyads found important to discuss with the nurses and to what extent all parts of the cognitive, supportive and behavioural components of the model were covered in the dialogues between dyads and nurses.

\section{Aim}

To describe nurses' documentation of the content in a psychoeducational intervention inspired by Stuifbergen's model addressing cognitive, supportive and behavioural needs of patient-partner dyads affected by HF.

\section{Design}

A descriptive, qualitative design was used to analyse nurses' documentation in dialogue guides used during sessions at the outpatient clinic with patients affected by $\mathrm{HF}$ and their partners. The study is part of a project aiming at developing and evaluating psychoeducational support to patient-partner dyads affected by HF.

ClinicalTrials.gov identifier: NCT02398799. ${ }^{20}$ 


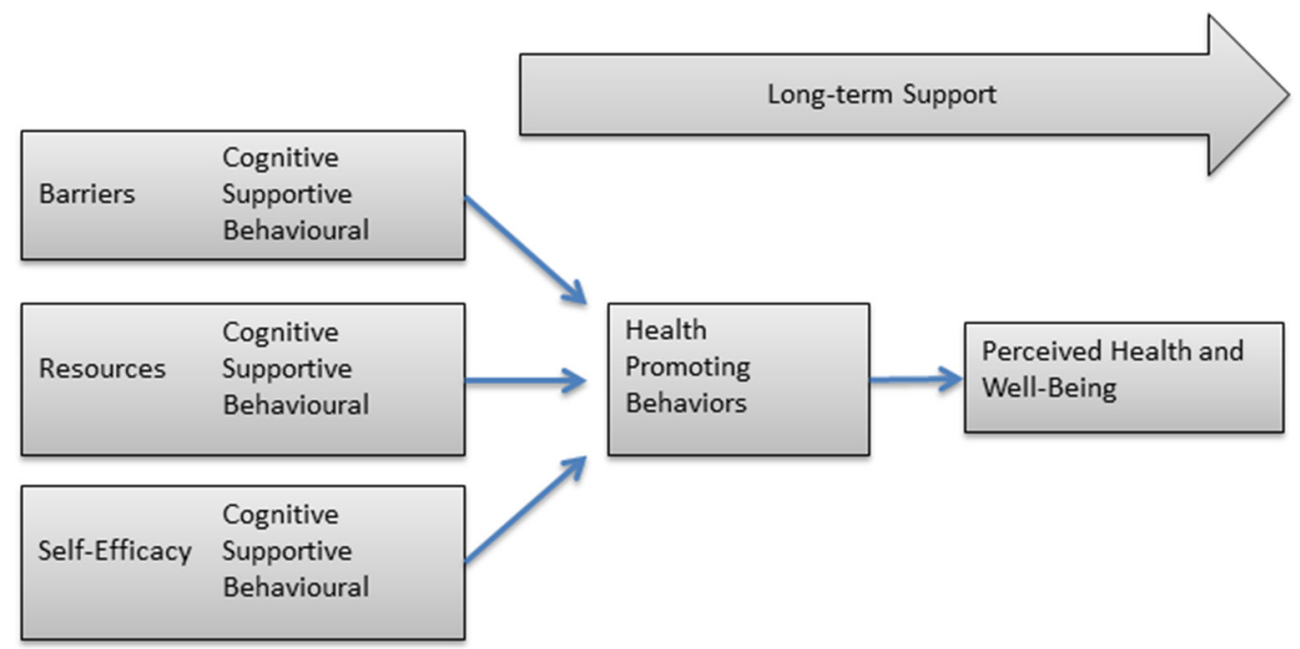

Figure 1 The conceptual health promotion model by Stuifbergen et al. ${ }^{22}$

\section{Procedure}

The research team started by constructing a dialogue guide whose theoretical framework was based on a conceptual health promotion model ${ }^{22} 26$ (figure 1). Previous interventions using the model have a cognitive component delivering information, but the major component focused on improving the patient's self-efficacy by identifying barriers, increasing support and developing skills and beliefs in one's capacity to achieve the desired behavioural changes.

In the current study, the dialogue guide included a cognitive, a supportive and a behavioural component and focused on helping patients and partners to change thoughts and behaviours and implement strategies for self-care management. All sessions included practical, educational and psychosocial topics, and the dyads were encouraged to speak about all subjects and concerns they felt they needed to discuss.

After construction, the five-page dialogue guide was used during three nursing sessions at the HF outpatient clinic. The sessions took place between years 2005 and 2008 and were scheduled 2, 6 and 12 weeks after patients were discharged from hospital after their admission due to worsening HF. Each session was performed as a discussion and focused on both the patients' and the partners' situation and experiences, their individual and mutual needs and expectations. All sessions included HF education to improve the dyads' problem-solving skills that would help them to recognise and modify factors contributing to psychological and emotional distress to maintain and strengthen their physical and mental functions and perceived control (figure 2). During the sessions, the

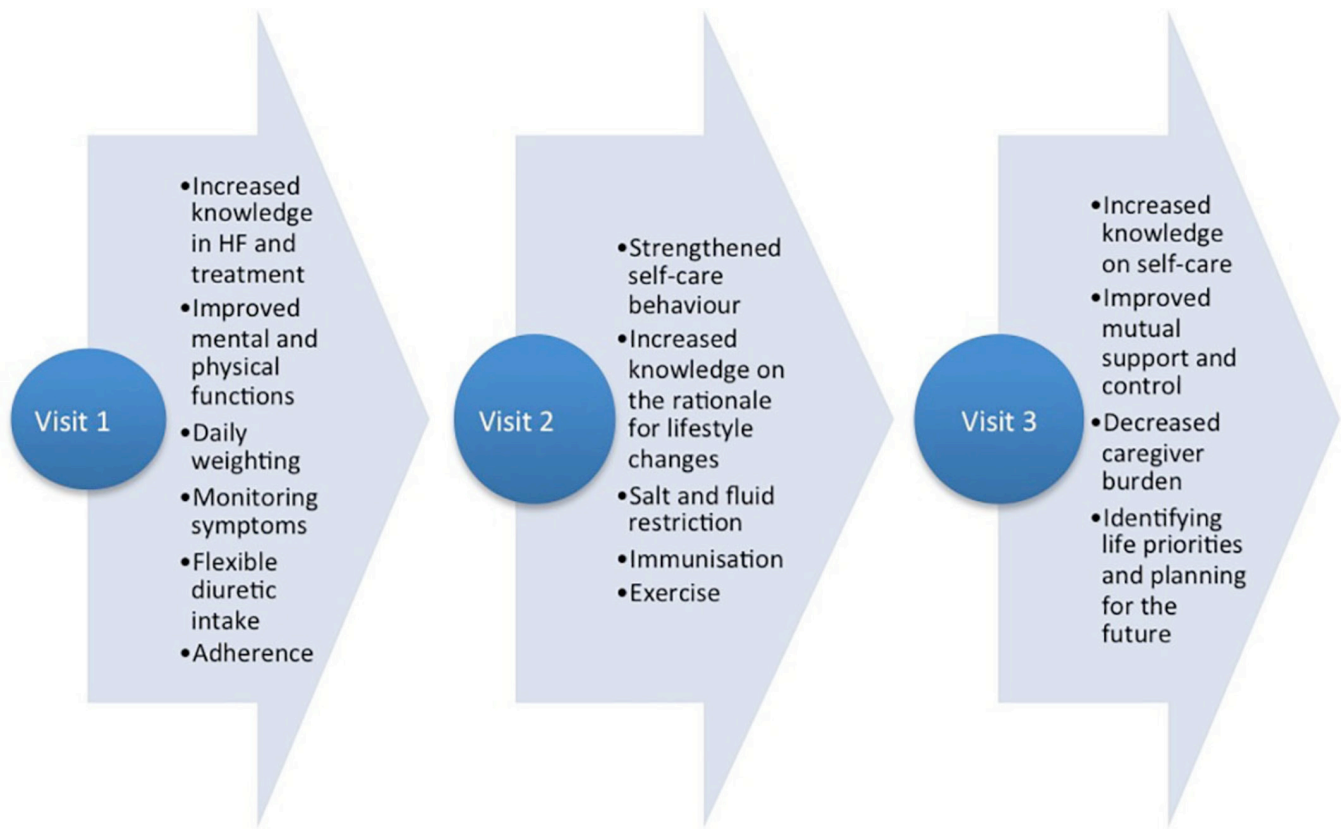

Figure 2 Cognitive, supportive and behavioural goals for each visit. 
Table 1 Characteristics of the participants

Patients ( $n=71)$ Partners $(n=71)$

\begin{tabular}{lll}
\hline Sociodemographics & & \\
Age, mean \pm SD & $69( \pm 14)$ & $67( \pm 12)$ \\
Female, $\mathrm{n}(\%)$ & $31 \%$ & $69 \%$ \\
NYHA class, $\mathrm{n}(\%)$ & & \\
II & $24(35)$ & \\
III & $40(55)$ & \\
IV & $7(10)$ &
\end{tabular}

Employment, n (\%)

\begin{tabular}{lll} 
Full time & $10(14)$ & $22(33)$ \\
$\begin{array}{l}\text { Disability pension/sick } \\
\text { leave }\end{array}$ & $13(17)$ & $2(3)$ \\
Home maker & $1(1)$ & $2(3)$ \\
Pension & $47(68)$ & $45(61)$ \\
\hline
\end{tabular}

NYHA, New York Heart Association.

patient and partner participated as equals and had the same opportunity to pose questions and speak about their individual concerns.

All four participating HF nurses were experienced in caring for HF patients and had attended 3 days of theoretical and practical training on how to deliver the intervention and document the dialogues before the study started. On several occasions, the study team visited the HF clinics and assessed the nurses' competence and study fidelity through observations and consultations. The study was designed in accordance with the World Medical Association Declaration of Helsinki and the Code of Ethics for Nurses. ${ }^{28}$ Permission was granted by the Regional Ethical Review Board in Linköping. The nurses and the dyads were informed that the documentation was planned to be used for research. The nurses all consented verbally that the material could be analysed. The dyads of patients and caregivers gave their informed consent to participating in the study and were aware that the dialogues were documented and planned to be analysed.

\section{Participants}

The dialogue guides from 71 patients with HF and their cohabiting partners were included in the analyses (table 1). The mean age for the patients was $69( \pm 14)$ years and partners $67( \pm 12)$ years, $69 \%$ of the patients were male and $55 \%$ were found to be in the New York Heart Association function class III. Regarding employment, $14 \%$ of the patients and $33 \%$ of the partners worked full time, $86 \%$ of the patients and $64 \%$ of the partners had a pension/were on sick leave, $3 \%$ of the partners were home makers.

\section{Data collection}

The data consisted of the nurses handwritten documentation in the five-page dialogue guides used during the intervention. During each of the three sessions, the nurses documented summaries of the discussions regarding the situation, given information and agreements and described difficulties.

\section{Data analysis}

A directed content analysis was selected for the analysis. ${ }^{29}$ Directed content analysis is by nature deductive and is used to validate or extend an existing theory or a model. The analysis is guided by a structured analytic process using existing research where the researcher begins with predetermined concepts as initial predetermined coding categories. ${ }^{30}$ These predetermined categories are then used to code the text. ${ }^{29}$ In the current study, we wanted to investigate to what extent Stuifbergen's model ${ }^{22}$ had been used and could be validated in the context, using the nurses' documentation.

The initial coding categories were based on the areas in the dialogue guide: (1) cognitive components; (2) supportive components and (3) behavioural components, which were developed from the conceptual health promotion model. ${ }^{22}$ The researchers started by developing operational definitions of the categories (see table 2).

Second, the first author (ML) typed out the content in the dialogue guides into a clear copy and numbered the transcripts so it was possible to see from which dialogue guide they were derived. To obtain a sense of the whole, transcriptions were read through several times by all authors. In the next step, the text was reviewed and text that described cognitive, supportive or behavioural components was sorted into one of the predetermined categories.

\section{Table 2 Coding framework based on the conceptual health promotion model}

\section{Coding category Description}

Cognitive component

Texts were coded into this category when statements described lifestyle modifications, medications and symptom management.

Supportive components

Texts were coded into this category when statements assessed the patient's or partner's need of support, partner's caregiver burden, modifying caregiver behaviour, strengthening self-care behaviour.

Behavioural components Texts were coded into this category when statements described barriers to lifestyle modifications, strategies to improve or maintain self-care behaviour, intentions, abilities and self-efficacy regarding self-care, planning for the future. 
The text in the dialogue guide was validated to be part of the predetermined categories or to be findings that were new and unknown. ${ }^{29} 31$ Each category was then reviewed and the content was divided into what were considered to be barriers, resources or self-efficacy, that is, the concepts in the model. Finally, we compared the extent to which the data were supported by Stuifbergen's model versus what represented new aspects not described in the model.

The first and the last author conducted the analysis, while the other two authors were involved in the discussions. Throughout the analysis, there was continuous back and forth movement between the whole text and the categories, including the concepts of the model, where all authors scrutinised and repeatedly discussed the results to ensure the trustworthiness of the data analysis.

\section{Rigour}

In qualitative research, the concepts of credibility, transferability, dependability and confirmability are considered aspects of trustworthiness. ${ }^{32}$

In this study, a directed deductive content analysis was chosen to validate an existing model..$^{22}$ Directed content analysis is a structured and systematic analysis process and the method guides the findings towards existing codes and categories. ${ }^{29}$ By using directed content analysis, trustworthiness can be achieved, as the text is compared with an existing model, which in turn has gone through a publication process. The systematic way of working with the analysis and the use of an existing model or theory strengthens the trustworthiness. ${ }^{33}$

To strengthen the credibility and confirmability, different strategies have been used.

The nurses' documentation in the dialogue guides has been used verbatim. The texts varied in depth and length. Some nurses wrote summatively and others described the discussions in more words. Nevertheless, all dialogue guides provided important and clear information on the components of dyad functioning as assessed during the sessions. The raw data consisted of handwritten text, but all nurses had clear handwriting so all data were credible.

The results have been discussed and compared with previous research. The authors are all experienced nurses and have worked with patients with HF for many years and also met their partners. During the analysis, the material has been read many times and the various components in the subcategories and the categories were reflected on, and this result can be seen as a possible interpretation of the data. An analyst triangulation was implied including both individual coding and consensus discussions in the research team allowing for testing negative cases and rival explanations.

To fulfil dependability, the authors maintained a decision trail developing the interview guide, during data collection, in analysis and also the research team regularly visited the nurses and assessed their competence to deliver the intervention through observations and consultations. Transferability of the findings was facilitated as the sample; the context and the analysis process are described in detail. Transferability to similar HF samples is possible since there were dialogue guides available from 71 dyads, which is a fairly large sample.

To ensure confidentiality, neither the names of the participants nor hospitals are published.

\section{Ethical considerations}

The study was approved by the Regional Ethical Review Board in Linköping, Sweden (Study code 03-568/M17804). The nurses at the HF clinic were informed that data would be analysed for study purposes.

\section{RESULTS}

The findings from the analysis showed that the conceptual health promotion model by Stuifbergen $e t a l^{22}$ was used during the psychoeducational sessions. The analysis confirmed the three predetermined categories: (1) cognitive components; (2) supportive components and (3) behavioural components. The content in the three categories was divided into the concepts barriers, resources and self-efficacy.

\section{Cognitive component}

Barriers

The major cognitive barrier for both patients and partners was the described lack of knowledge. Some patients had received information prior to the sessions but several patients had no prior knowledge about the disease. They did not know that self-care could be carried out and affect their well-being although they had lived with the diagnosis for many years. Others described that they did not really understand that they were sick and the lack of insight had meant that they had not engaged in self-care activities.

Almost none of the partners expressed that they had prior knowledge and therefore they had not been involved in the patients' self-care activities. A few partners had searched for information about HF on the internet but found many websites were lacking practical information which became more of a cognitive barrier than a resource in daily living.

As they discussed their expectations of the sessions, the dyads expressed a broad range of expectations varying from a wish and belief that the patient would be cured to the other side of the coin; that it does not matter what we do because both body and heart are too old and too sick. Also a few partners did not want to know anything about HF because it made them feel worried and uneasy.

\section{Resources}

Cognitive resources were found when the dyads expressed a wish to learn about HF. During the first session, both patients and partners posed general questions about HF treatment, causes and prognosis after receiving a general knowledge overview from the nurse. However, with increased knowledge, both patients' and partners' questions were more specific regarding how to perform self-care activities on a daily basis. 


\section{Self-efficacy}

During the sessions, the dyads described that they had enhanced their knowledge and had greater confidence to handle their situation. The patients felt they have had an opportunity to pose questions and now had in-depth knowledge of the disease and, due to that, the confidence to act had increased.

Partners felt they had received guidance regarding exercise, for example how much someone who has HF can walk without it becoming harmful. So now they encouraged the patient to exercise. They also learnt about and felt confident in how to act in case of deterioration and how deterioration could be prevented.

\section{Supportive component \\ Barrier}

Supportive barriers could be both emotional and practical, and both patients and partners described that they often lacked support. Examples of emotional barriers were that due to anxiety and consideration, some partners tried to set limits to physical activity so patients did not overstrain themselves. When patients felt overprotected, this sometimes led to tension and conflicts in the relationship. During the discussions, the nurses helped and guided dyads to communicate their thoughts and concerns with each other.

Another barrier was loss of friends' due to the disease. Patients could no longer socialise as before and therefore the partner had less social support, activities and interactions. The dyads became isolated since the partner did not want to leave patient alone and do things on their own.

Dyads described a lack of good healthcare contacts. Nurses' documentation revealed that many patients were sad and depressed over their situation. Partners described a worry and asked for long-term guidance from healthcare professionals to learn how to offer support. Since several partners had no previous knowledge about the disease, the sessions brought up many thoughts that they needed the nurses' support to process.

The dyads expressed a wish for further, regular visits at the HF outpatient clinic. The visits had created security for both patient and partner and they expressed anxiety when the possibly to contact the nurses would end. The visits had made them feel secure and they knew they had someone they could contact when questions arose.

A practical, supportive barrier was if the partner also had some illness and therefore was not able to provide support to the patient with HF and maybe instead needed help and support themselves. Need of formal support from someone outside the family arose primarily if both patient and partner had difficulty carrying out practical tasks, such as driving or managing the household.

Some dyads experienced hopelessness when the future was discussed and patients were perceived as depressed in the nurses' documentation. Some patients expressed a need for contact with a social worker; others were in continuous need of support from homecare.
Resources

Many dyads said that they helped each other and shared the chores on the basis of strength and ability. With increased knowledge, they seemed to understand better why it is important to perform self-care activities, and for some patients this led to a need of support from the partner. Partners wished to learn and become more involved in the care and help with decision-making on a daily basis.

The need for support increased when patients were depressed or suffered from multimorbidity. Some patients had previously had contact with a social worker and have, due to these visits, been helped to accept their situation.

\section{Self-efficacy}

The documentation described that many partners give plenty of practical support but also how they encouraged patients to take own responsibility. Also, when the partner had knowledge and the patient was motivated, confidence in their own abilities increased in both patients and partners.

\section{Behavioural component \\ Barrier}

A behavioural barrier was patients' lack of strength, which led to involuntary inactivity. Both patients and partners felt that partners had to take on more of the workload in the household. The additional burden was described as mentally exhausting for the partners due to a greater responsibility.

Some dyads described that the new roles led to conflicts and fractions in the relationship. Partners thought it was difficult to leave the patient alone and that they had to hurry home from work, leaving no time for own activities.

Some patients found it hard to follow dietary advice, to weigh themselves daily and to monitor and assess HF signs and symptoms. For some dyads, HF had been a barrier for sexuality, where the patient no longer has the energy and the partner no longer dares to have a sexual relation. The documentation did not reveal how the nurse discussed the perceived sexual difficulties with the dyads.

\section{Resources}

Most patients wished to take the main responsibility to manage their self-care, but sometimes were lacking confidence in their own ability and doubting the effect of selfcare advices. When seeing the effects of their self-care activities, the patients' confidence to manage self-care increased.

For some dyads, the new roles had strengthened the relationship. They collaborated to follow instructions about behavioural changes and provided mutual support to each other. In some relationships, the partner functioned as a resource for the patient, someone to discuss strategies with. Other partners provided help with selfcare on a daily basis. 


\section{Self-efficacy}

During the last visit, the nurses assessed that most of the dyads had a good understanding about HF. They had confidence when following the self-care advice given during the sessions and had gradually adapted to the situation. Adaptations could, for example, be a change of accommodation or partner's employment.

\section{DISCUSSION}

Our study showed that the model developed by Stuifbergen et $a l^{22}$ covering cognitive, supportive and behavioural components of dyad functioning, was fully addressed during the nursing sessions. The findings revealed a wide range of cognitive, supportive and behavioural needs among the dyads. The model highlights the importance of identifying and discussing barriers, resources and self-efficacy to increase the dyads health-promoting behaviours.

For some dyads, the new roles had strengthened their relationship; patients and partners collaborated and provided mutual support to each other. It is known that supporting a person with HF involves several salient changes in daily life and includes both benefits and burdens. It represents an opportunity for increased intimacy with the person who is ill. Caregiving is sometimes experienced as rewarding and provides satisfaction and hopes for a positive future. ${ }^{3435}$

The nurses' documentation showed that most patients found great support in their partners and partners provided both practical and emotional support. Caregiver support is known to improve self-care and strengthen patients' adherence to medical treatment and maintaining a healthy lifestyle. ${ }^{3637}$ However, one recent study found the contrary. Cocchieri and colleagues ${ }^{38}$ found that having a caregiver was associated with poor self-care maintenance. They concluded that their result might be due to the fact that patients having a partner were more cognitive and functionally impaired, had higher comorbidity and were in need of more care.

Caregiving may sometimes be experienced as burdensome for the partner. Partners in the current study described their having taken over much of the household workload and helped the patients with practical tasks regarding the patient's self-care management and for some it meant a burden. One third of partners of patients with HF perceive moderate caregiver burden and could be at higher risk of worsened health. ${ }^{11}$ As in previous research, partners sacrificed their own needs to support care for the patient. Partners perceived a need for support with daily living as well as easy access to the healthcare providers. ${ }^{39}$ To reduce caregiver burden, interventions should focus on improving social support and provide knowledge of HF to both patients and caregivers to increase their perceived control over the situation. ${ }^{34}$

With the increasing awareness of patient needs, there are a lot of educational moments in most current $\mathrm{HF}$ care. However, it still seems important to consider cognitive aspects during nursing sessions. Patients felt that they had never received information about their illness previously. One reason could be that they had suffered from HF for many years and only received education at the time of the diagnosis, and now the information has been forgotten. Another reason could be that there is a high occurrence of mild cognitive deficits among patients with $\mathrm{HF}$ without known cognitive diseases or disorders. ${ }^{40}$ This calls for repeated information and follow-ups, and close attention should be paid to the patient's self-care abilities and compliance, as inadequate self-care activities could lead to repeated hospitalisations. Cognitive impairment presents a barrier to the complex medication and self-care management that is required in HF treatment. ${ }^{41}$ Due to this, both patients and caregivers perceive a need to have a long-term healthcare contact they can turn to when questions arise. ${ }^{39} 42$ This is supported in the present results where the dyads expressed a need for long-term support from nurses, and for some, also homecare and social workers.

The behavioural component includes self-care abilities, and a prerequisite for self-care adherence is to gain knowledge. Self-care has been defined as a naturalistic decision-making process which influences actions that maintain physiological stability, facilitates the perception of symptoms and directs the management of symptoms. ${ }^{43}$ Patients and partners need to learn about HF to become active participants in the management of the illness but sometimes their own knowledge was not enough and then the dyads wanted easy access to healthcare. Long-term support was highlighted as an important factor to remain being perceived as healthy and experiencing well-being. Previous research has also addressed both patients' and caregivers' need for a regular healthcare contact they can easily contact, as they currently experience difficulties both navigating for and assessing support. ${ }^{44}$ Today, a lot of different healthcare professionals (primary care physicians and nurses, cardiologists and HF nurses) could be involved in the care for HF patients. Patients and caregivers have to decide who to contact based on their previous experiences, which is perceived as confusing and leads to insecurity. Instead, to have a permanent healthcare contact they can turn to if necessary would facilitate daily life.

\section{Limitations}

In qualitative research, generalisability of the findings may be restricted to a particular setting or context. However, the current study findings are consistent with previous research using the model ${ }^{22}$ in a different setting and for patients with MS.

The nurses were informed beforehand that their documentation was going to be analysed and the documentation in the dialogue guides was transcribed verbatim. The text varied in depth and length. Some nurses wrote summatively and others described the discussions in more words. Nevertheless, all dialogue guides were found to provide important and clear information on the components of dyad functioning as assessed during the sessions. 
The raw data consisted of handwritten text which may affect credibility. However, all nurses had clear handwriting so all data were considered accurate.

The data were collected between the years 2005 and 2008. There have been some changes in HF treatment since then; however, the importance of self-care and family support has been increasingly acknowledged in guidelines, ${ }^{1}$ thus making this study even more relevant today than when the data were collected. Further, since the aim of the study was to describe nurses' documentation regarding a psychoeducational intervention inspired by a health promotion model, the data are still valid and of interest. To date, there are still very few models or theory-based interventions for patients and partners living with $\mathrm{HF}_{1}{ }^{175}$ and our results can hopefully serve as an example and inspiration for further research in this area.

\section{CONCLUSION}

This study described how cognitive, supportive and behavioural components of dyad functioning were addressed during nursing sessions with dyads affected by HF.

We found the model to be suitable for helping patientpartner HF dyads to gain knowledge and develop skills to handle living with HF. The model promoted healthy and supportive behaviours that is to improve self-care. The results show the importance of providing continuous healthcare contacts throughout the illness trajectory. The findings emphasise that gaining knowledge of HF is not enough. It also seems clear that one type of intervention does not fit all dyads, as a variety of equal and diverse needs emerged during the analysis, and when the needs were met the dyads developed self-efficacy. It is important that healthcare providers take time to discuss all aspects related to the disease that are of importance for the dyad to support improved self-efficacy and reduce insecurity. Furthermore, the model was applicable for dyads consisting of a patient with $\mathrm{HF}$ and their partner, not only for patients as shown in previous studies implementing the model.

The result can inspire others when using this model or designing new psychoeducational dyad interventions. The findings provide practical examples of cognitive, supportive and behavioural needs, which need to be considered when meeting patients and partners affected by HF. This knowledge can be applied in HF units to develop a dyad educational regimen.

\section{Twitter@MartorMaria}

Acknowledgements We would like to thank all participating patients, partners and heart failure nurses who participated in the study. We also thank David Strömberg for language editing.

Contributors SÅ and ML collected the data. ML was mainly responsible for analysing data and drafting the manuscript and $S \AA$, TJ and AS contributed to the data analysis and writing the manuscript. AS and TJ conceptualised and developed the idea for the study as part of the broader research programme. All authors critically edited the manuscript and read and approved the final version.

Competing interests None declared.
Patient consent Obtained.

Ethics approval Regional Ethical Review Board in Linköping, Sweden (Study code 03-568/ M178-04).

Provenance and peer review Not commissioned; externally peer reviewed.

Data sharing statement № additional data are available.

Open Access This is an Open Access article distributed in accordance with the Creative Commons Attribution Non Commercial (CC BY-NC 4.0) license, which permits others to distribute, remix, adapt, build upon this work non-commercially, and license their derivative works on different terms, provided the original work is properly cited and the use is non-commercial. See: http://creativecommons.org/ licenses/by-nc/4.0/

(C) Article author(s) (or their employer(s) unless otherwise stated in the text of the article) 2017. All rights reserved. No commercial use is permitted unless otherwise expressly granted.

\section{REFERENCES}

1. Ponikowski $P$, et al. ESC Guidelines for the diagnosis and treatment of acute and chronic heart failure: The task force for the diagnosis and treatment of acute and chronic heart failure of the European Society of Cardiology (ESC). Developed with the special contribution of the Heart Failure Association (HFA) of the ESC. Eur J Heart Fail 2016.

2. Riegel B, Moser DK, Anker SD, et al. State of the science: promoting self-care in persons with heart failure: a scientific statement from the American Heart Association. Circulation 2009;120:1141-63.

3. Buck HG, Harkness K, Wion R, et al. Caregivers' contributions to heart failure self-care: a systematic review. Eur $J$ Cardiovasc Nurs 2015;14:79-89.

4. Clark AM, Spaling M, Harkness K, et al. Determinants of effective heart failure self-care: a systematic review of patients' and caregivers' perceptions. Heart 2014;100:716-21.

5. Molloy GJ, Johnston DW, Witham MD. Family caregiving and congestive heart failure. Review and analysis. Eur J Heart Fail 2005;7:592-603.

6. Dinesen B, Nøhr C, Andersen SK, et al. Under surveillance, yet looked after: telehomecare as viewed by patients and their spouse/ partners. Eur J Cardiovasc Nurs 2008;7:239-46.

7. Lamura G, Mnich E, Nolan M, et al. Family carers' experiences using support services in Europe: empirical evidence from the EUROFAMCARE study. Gerontologist 2008;48:752-71.

8. Daugherty J, Saarmann L, Riegel B, et al. Can we talk? Developing a social support nursing intervention for couples. Clin Nurse Spec 2002;16:211-8.

9. Ohman M, Söderberg S. The experiences of close relatives living with a person with serious chronic illness. Qual Health Res 2004;14:396-410.

10. Rees J, O'Boyle C, MacDonagh R. Quality of life: impact of chronic illness on the partner. J R Soc Med 2001;94:563-6.

11. Agren S, Evangelista L, Strömberg A. Do partners of patients with chronic heart failure experience caregiver burden? Eur J Cardiovasc Nurs 2010;9:254-62.

12. Luttik ML, Jaarsma T, Veeger $\mathrm{N}$, et al. Caregiver burden in partners of Heart Failure patients; limited influence of disease severity. Eur $J$ Heart Fail 2007;9(6-7):695-701.

13. Dunbar SB, Clark PC, Deaton C, et al. Family education and support interventions in heart failure: a pilot study. Nurs Res 2005;54:158-66.

14. Strömberg A. The crucial role of patient education in heart failure. Eur J Heart Fail 2005;7:363-9.

15. Srisuk N, Cameron J, Ski CF, et al. Heart failure family-based education: a systematic review. Patient Educ Couns 2016;99:326-38.

16. Evangelista LS, Strömberg A, Dionne-Odom JN. An integrated review of interventions to improve psychological outcomes in caregivers of patients with heart failure. Curr Opin Support Palliat Care 2016;10:24-31.

17. Dunbar SB, Clark PC, Reilly CM, et al. A trial of family partnership and education interventions in heart failure. J Card Fail 2013;19:829-41.

18. Löfvenmark C, Saboonchi F, Edner M, et al. Evaluation of an educational programme for family members of patients living with heart failure: a randomised controlled trial. J Clin Nurs 2013;22(12):115-26.

19. Etemadifar S, Bahrami M, Shahriari M, et al. The effectiveness of a supportive educative group intervention on family caregiver burden of patients with heart failure. Iran J Nurs Midwifery Res 2014;19:217-23. 
20. Liljeroos M, Ågren S, Jaarsma T, et al. Long term follow-up after a randomized integrated educational and psychosocial intervention in patient-partner dyads affected by heart failure. PLoS One 2015;10:e0138058.

21. Ryan RM, Deci EL. Self-determination theory and the facilitation of intrinsic motivation, social development, and well-being. Am Psychol 2000;55:68-78.

22. Stuifbergen A, Becker H, Rogers S, et al. Promoting wellness for women with multiple sclerosis. J Neurosci Nurs 1999;31:73-9.

23. Pender NJ, Pender AR. Health promotion in nursing practice. 2nd ed. Norwalk, Conn: Appleton \& Lange, 1987.

24. Bandura A. Self-efficacy: toward a unifying theory of behavioral change. Psychol Rev 1977;84:191-215.

25. Bandura A. Self-efficacy: The exercise of control. New York: W.H. Freeman, 1997.

26. Stuifbergen AK, Becker $\mathrm{H}$, Blozis $\mathrm{S}$, et al. A randomized clinical trial of a wellness intervention for women with multiple sclerosis. Arch Phys Med Rehabil 2003;84:467-76.

27. Revenson TA, et al. Caregiving in the illness context. 2016.

28. World Medical Association. World Medical Association Declaration of Helsinki: ethical principles for medical research involving human subjects. JAMA 2013;310:2191-4.

29. Hsieh HF, Shannon SE. Three approaches to qualitative content analysis. Qual Health Res 2005;15:1277-88.

30. Potter WJ, Levine-Donnerstein D. Rethinking validity and reliability in content analysis. Journal of Applied Communication Research 1999;27:258-84.

31. Elo S, Kyngäs $\mathrm{H}$. The qualitative content analysis process. J Adv Nurs 2008;62:107-15.

32. Patton MQ. Qualitative research \& evaluation methods. London: SAGE, 2002.

33. Lincoln Y, Guba E. Naturalistic inquiry. Newbury Park, London: Sage Publications Inc, 1985.
34. Strömberg A. The situation of caregivers in heart failure and their role in improving patient outcomes. Curr Heart Fail Rep 2013;10:270-5.

35. Duggleby W, Holtslander L, Kylma J, et al. Metasynthesis of the hope experience of family caregivers of persons with chronic illness. Qual Health Res 2010;20:148-58.

36. Gallagher R, Luttik ML, Jaarsma T. Social support and self-care in heart failure. J Cardiovasc Nurs 2011;26:439-45.

37. Pressler SJ, Gradus-Pizlo I, Chubinski SD, et al. Family caregivers of patients with heart failure: a longitudinal study. J Cardiovasc Nurs 2013;28:417-28.

38. Cocchieri A, Riegel B, D'Agostino F, et al. Describing self-care in Italian adults with heart failure and identifying determinants of poor self-care. Eur J Cardiovasc Nurs 2015;14:126-36.

39. Doherty LC, Fitzsimons D, Mcllfatrick SJ. Carers' needs in advanced heart failure: A systematic narrative review. Eur J Cardiovasc Nurs 2016;15:203-12.

40. Nordlund A, Berggren J, Holmström A, et al. Frequent mild cognitive deficits in several functional domains in elderly patients with heart failure without known cognitive disorders. J Card Fail 2015;21:702-7.

41. Cannon JA, McMurray JJ, Quinn TJ. 'Hearts and minds': association, causation and implication of cognitive impairment in heart failure. Alzheimers Res Ther 2015;7:22.

42. Liljeroos $\mathrm{M}$, Agren $\mathrm{S}$, Jaarsma $\mathrm{T}$, et al. Perceived caring needs in patient-partner dyads affected by heart failure: a qualitative study. $J$ Clin Nurs 2014;23:2928-38.

43. Riegel B, Dickson VV, Faulkner KM. The situation-specific theory of heart failure self-care: revised and updated. J Cardiovasc Nurs 2016;31:226-35

44. Browne S, Macdonald S, May CR, et al. Patient, carer and professional perspectives on barriers and facilitators to quality care in advanced heart failure. PLoS One 2014;9:e93288.

45. Sebern MD, Woda A. Shared care dyadic intervention: outcome patterns for heart failure care partners. West $J$ Nurs Res 2012;34:289-316. 